\title{
Isolated muscle hypertrophy as a sign of radicular or peripheral nerve injury
}

\author{
Heinrich P Mattle, Christian W Hess, Hans-Peter Ludin, Marco Mumenthaler
}

\begin{abstract}
Two patients with isolated neurogenic hypertrophy of the trapezius muscle due to accessory nerve injury and a patient with neurogenic hypertrophy of the anterior tibial muscle due to chronic radicular lesion $L 4$ are described. Electromyography of the affected muscles showed dense continuing spontaneous discharges of complex potentials. Muscle biopsy performed in two patients showed abundant hypertrophic muscle fibres, identified in one case by ATP-ase reaction as being of predominantly type $I$. In the majority of previously reported patients with neurogenic muscle hypertrophy confined to the calf muscle, a passive stretch mechanism was suggested as a cause of the hypertrophy. It is assumed that the excessive spontaneous muscle activity gave rise to the hypertrophy in these patients. This may also be true in previously reported patients with neurogenic hypertrophy and similar spontaneous activity in electromyography.
\end{abstract}

Neurogenic lesions normally cause muscle wasting. But occasionally a neurogenic lesion results in hypertrophy of a single muscle. In $1848 \mathrm{R} J$ Graves reported a man with sciatica and subsequent calf enlargement. ${ }^{1}$ A second case was published in France: in 1918 Lhermitte noticed a man with calf enlargement after a bullet injury to the sciatic nerve. ${ }^{2}$ In 1932 he reported a second ${ }^{3}$ and a third patient with the same enlargement after sciatica. ${ }^{4}$ Further cases of calf enlargement after sciatica were published later. ${ }^{5-17}$. Both Valenstein et $a l^{18}$ and Vasilescu et $a l^{19}$ described calf enlargement with chronic relapsing polyneuropathy. In his first report ${ }^{2}$ Lhermitte also commented on a soldier who experienced a thenar hypertrophy after a forearm injury, and neurogenic hypertrophy of the hand and forearm muscles with chronic relapsing inflammatory polyneuropathy has been described in a recent report. ${ }^{16}$

Our three patients illustrate that neurogenic hypertrophy of muscles due to radicular or peripheral nerve lesions is not restricted to the calves and may also affect the trapezius and anterior tibial muscles. They suggest a common pathogenetic pathway for neurogenic muscle hypertrophy.

\section{Case reports}

Patient 1 This 39 year old athletic man was involved in a head-on car collison nine years previously. He was wearing a seat belt and suffered only minor injuries. Subsequently he noticed increasingly painless wave-like muscle motions above his right shoulder. There was no weakness or sensory disturbance. The right trapezius muscle bulk gradually increased, and because of two episodes of torticollis the patient sought medical advice.

The family history for muscle or nerve disease was negative. The clinical examination showed normal cranial nerves, normal neck mobility, and symmetrical tendon jerks. The Babinski sign was negative. Motor and sensory functions, coordination, and gait were normal. Sweating was symmetrical. There was increased muscle bulk of his right trapezius muscle (fig 1) and frequent muscle twitching. General physical examination was normal. Laboratory tests such as erythrocyte sedimentation rate (ESR), white cell count (WCC), serum chemistries including glucose and calcium, and creatine phosphokinase (CPK) were unremarkable. Radiographs of the chest, shoulders, and scapulae and EKG were normal. CT (fig 2) of the cervico-thoracic region confirmed an increased size and decreased density of the right trapezius muscle.

Electrophysiological studies: electromyography of the right trapezius muscle showed continuous spontaneous activity at all sites as numerous mostly polyphasic and large potentials (peak to peak amplitudes of up to $1.2 \mathrm{mV}$ )

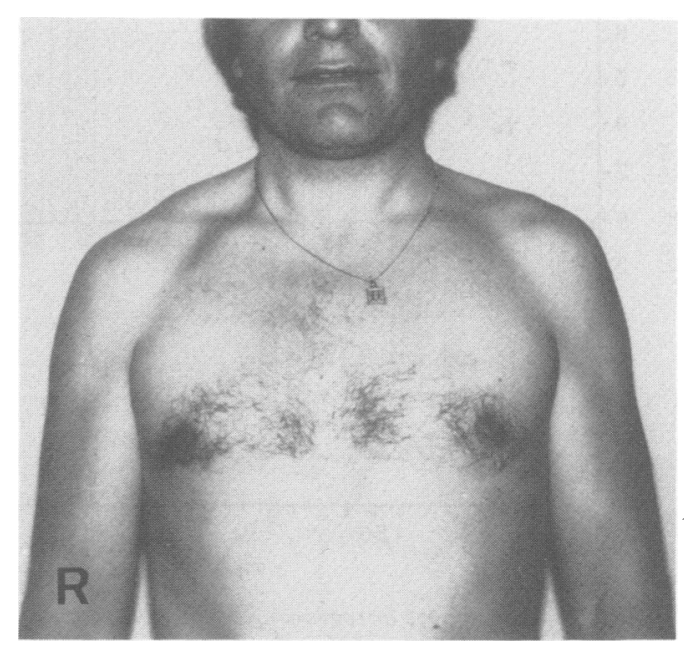

Figure 1 Patient 1 presenting with a hypertrophied right trapezius muscle $(R=$ right $)$. 


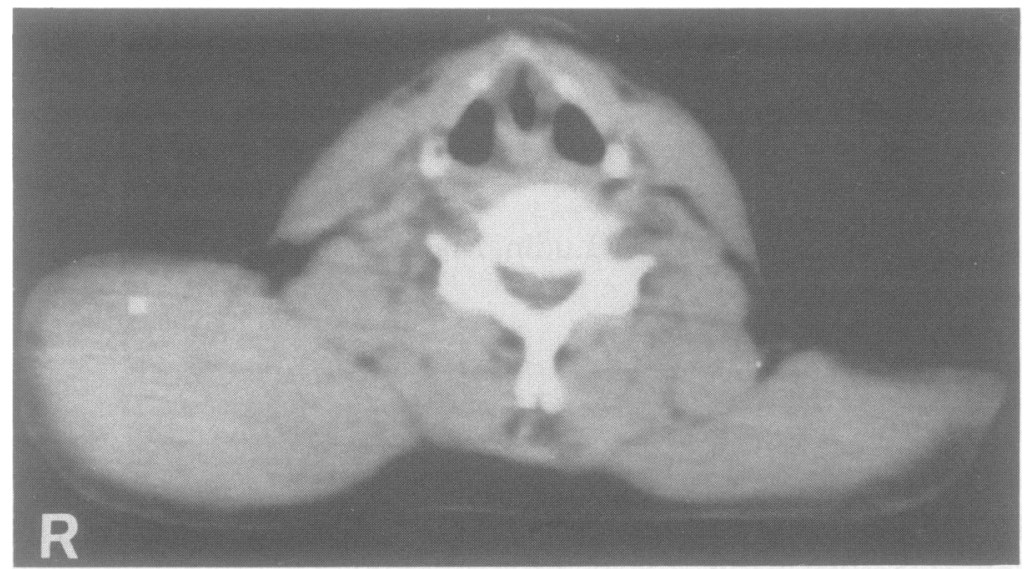

Figure $2 C T$ scan of an axial section through the trapezius muscles of patient 1. Note the enlarged right trapezius muscle compared with the normal left side $(R=$ right $)$.

occurring at random or as brief grouped discharges. The interference pattern during maximal voluntary effort was normal. Motor unit potential analysis revealed a moderate prolongation of the average potential duration $(18.6 \mathrm{~ms}, 23$ potentials measured). Electromyography of the right sternocleidomastoid muscle was normal. Both accessory nerves showed normal motor latencies to the trapezius muscles.

Muscle biopsy from the right trapezius showed sections with abundant hypertrophic fibres predominantly of type I as well as sections with small groups of atrophic, elongated type II fibres according to the ATP-ase reaction, and type grouping was evident (figs 3,4 ). The overall distribution of type I and II fibres was normal. A diagnosis of right accessory nerve lesion was made.

Patient 2 The family history of this 20 year old asthenic man was negative. At the age of 18 he had a scalenic lymph node biopsy on his left side. The lymph node histology was compatible with a cat scratch disease. Approximately one month later he felt pain in his left shoulder and subsequently noticed weakness of his left shoulder and arm. He was referred six

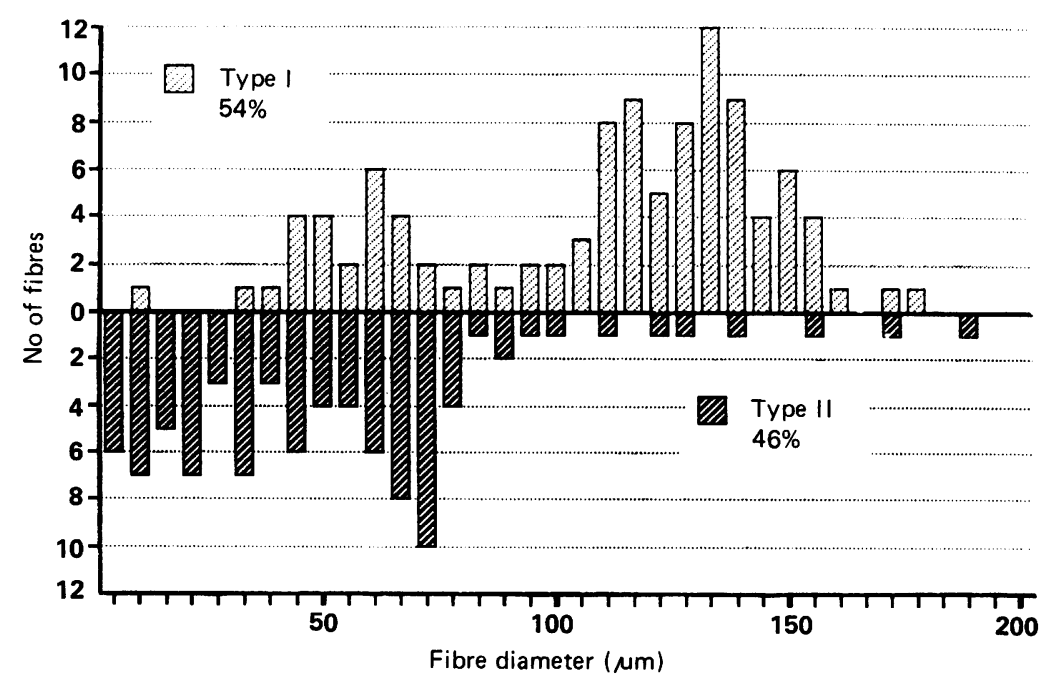

Figure 3 Muscle fibre histogram of the biopsy from the trapezius muscles of patient 1 showing fibre diameters and type distribution. There are abundant hypertrophic fibres, predominantly of type I and also atrophic type II fibres. Fibre type distribution is normal (Specimen 860916, ATP-ase, pH 4.6, $10 \times$ ).

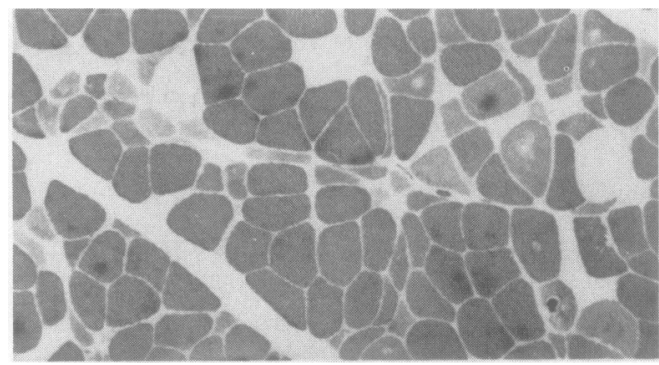

Figure 4 Biopsy from the same specimen as in fig 3 showing a sample with groups of atrophic, angulated fibres, all belonging to type II. Type grouping and targetoid fibres are also present (ATP-ase, pH 4.6, $7 \times)$.

months after the lymph node biopsy. Except for the atrophy of the upper part of his left trapezius muscle, the general physical and neurological examinations were normal. Bulk and function of the sternocleidomastoid muscle was normal. ESR, WCC, CPK, and radiographs of the shoulder were normal. The clinical diagnosis was a left accessory nerve lesion and it was corroborated by electromyography.

As there was no spontaneous improvement, the patient had surgery for his nerve lesion. The surgical findings showed a completely transsected left accessory nerve at the site of the lymph node biopsy. The proximal and distal nerve endings were easily found, an anastomosis was made, and the nerve was sutured. Six months later electromyography showed "high frequency complex repetitive discharges" in the left trapezius muscle. A year later there was a prominent hypertrophy of the left trapezius muscle. The muscle weakness was still present, with force against very slight resistance (Grade 3 to 4 according to the MRC scale).

Electromyography of the upper portion of the left trapezius muscle showed continuous and dense repetitive discharges of simple or polyphasic potentials mostly of high frequency $(>10 \mathrm{~Hz})$ at all sites in the completely relaxed muscle. Additional discharges were triggered by needle movements and voluntary contractions. There was a fair amount of voluntary activity, which yielded an intermediate pattern at maximal voluntary activation. Stimulation of the left accessory nerve produced a muscle response of prolonged latency in the trapezius muscle. A diagnosis of left accessory nerve lesion was made.

Patient 3 This was a 41 year old farmer and ski instructor with a negative family history for muscle and nerve diseases. At the age of 29 he

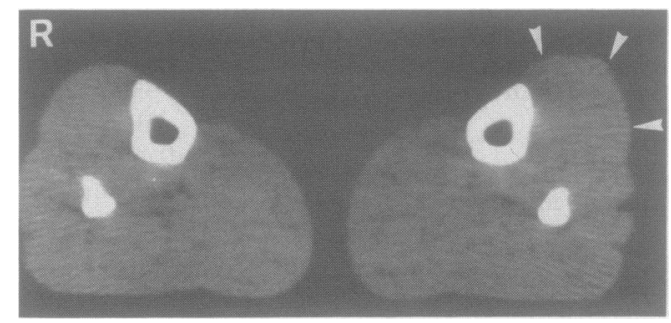

Figure 5 CT scan through the lower legs of patient 3. Note the hypertrophic left anterior tibial muscle (arrowheads) compared with the normal right side $(R=$ right $)$. 
Figure 6 Needle-EMG from the left tibialis anterior muscle of patient 3 showing continuous slow frequency rhythmic repetitive activity of complex potentials typical of "myokymic discharges".
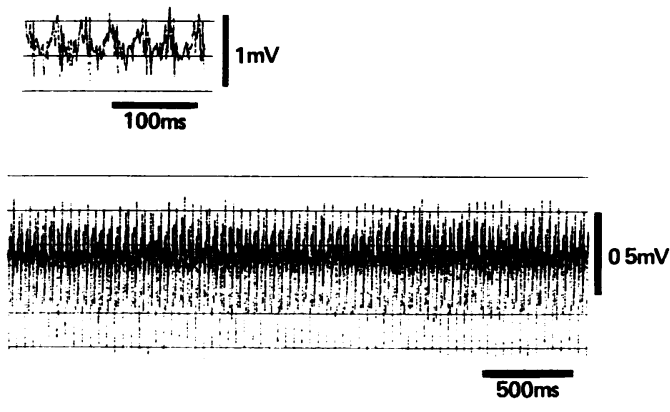

had an operation for a left lumbar $3 / 4$ disc hernia and a spondylodesis. In the following years he had intermittent back pain radiating to his left thigh distally and medially and to his lower leg anteriorly and medially. Nonsteroidal anti-inflammatory drugs reduced the pain. At the age of 40 , however, he experienced a substantial increase in discomfort, and for the first time noticed a gradually increasing swelling, lateral to his left tibia. Suspecting a muscle sarcoma, a biopsy was taken and was considered negative. CT showed an increased bulk of the anterior tibial muscle with a normal density (fig 5). One year later he was referred for further investigation since the muscle swelling was still increasing.

The clinical examination showed a painless swelling of the left anterior tibial muscle. Elevation of the left foot was slightly weaker than on the right side. The left knee jerk was decreased. The remaining tendon jerks were symmetric and brisk. The circumference of the thighs was equal, but the left lower leg measured $34 \mathrm{~cm}$ and the right only $32 \mathrm{~cm}$. There were no other neurological abnormalities. Skin temperature was $32^{\circ} \mathrm{C}$ over both anterior tibial muscles ruling out an inflammatory process. ESR, WCC, electrolytes, glucose, creatinine, serum aspartate aminotransferase, alkaline phosphatase, and chest radiographs were normal. Serum alanine aminotransferase was $46 \mathrm{mU} / \mathrm{ml}$ (normal up to $27 \mathrm{mU} / \mathrm{ml}$ ) and CPK was $159 \mathrm{mU} / \mathrm{ml}$ (normal up to $125 \mathrm{mU} / \mathrm{ml}$ ).

The muscle biopsy specimens obtained a year earlier in another laboratory were revised. Only $\mathrm{HE}$ and van Gieson stains were available. The fibre size showed an abnormal variability. Hypertrophic fibres were present in large number, some with segmentation and an increased number of central nuclei. The aspect was non-specific and was considered consistent with both a myopathic process and with secondary myopathic changes due to a chronic denervation.

Electromyography of the left anterior tibial muscle showed continuing high and low frequency repetitive discharges of mostly polyphasic potentials at all sites in the relaxed muscle (fig 6). In addition, there were grouped discharges of regularly occurring bursts of several potentials. The density of this spontaneous activity obscured the motor unit potentials of volitional activation at the optical signal display. The left gastrocnemius muscle showed a reduced pattern at maximal voluntary activity but no spontaneous activity. Electromyography of the left lateral vastus muscle was normal. The motor conduction velocity of the left peroneal nerve was normal over all segments.

A diagnosis of muscle hypertrophy due to longstanding left L4 root lesion was made.

\section{Discussion}

Hypertrophy is a physiological response of muscle to work. ${ }^{2021}$ Stretch may also induce muscle hypertrophy, ${ }^{22}$ even in the absence of innervation. Pathological conditions leading to muscle hypertrophy include myotonia congenita and other forms of myotonias, ${ }^{23}$ dystonias, acromegaly, chronic spinal muscle atrophy, ${ }^{24}{ }^{26}$ familial ${ }^{27}$ and a sporadic ataxia, ${ }^{28}$ the Schwartz-Jampel syndrome, ${ }^{29} 30$ the Hoffmann's syndrome, ${ }^{31}$ post-poliomyelitis muscle hypertrophy, ${ }^{32}$ and polyneuropathy associated with continuous spontaneous activity. ${ }^{18} 1933-35$ Muscle hypertrophy following nerve injury has been the subject of experimental work. ${ }^{36}$ The phenomenon has been reported occasionally in humans ${ }^{1-17}$ but rarely cited in textbooks. ${ }^{37}$

All reported human cases of muscle hypertrophy due to radicular or peripheral nerve injuries were restricted to the calves except one of Lhermitte's cases. ${ }^{2}$ Bernat and Ochoa proposed combined work- and stretch-induced post-denervation muscle hypertrophy as an explanation. ${ }^{8}$ They supported their hypothesis with biopsy findings from the gastrocnemius muscle. These showed changes of partial denervation and reinnervation, with small groups of type I and II atrophic muscle fibres and abundant hypertrophic fibres of both types but mostly type II. They postulated that, in addition to compensatory work-induced type II muscle fibre hypertrophy, there was an element of stretch-induced type I hypertrophy of denervated fibres. Such a condition has been well recognised experimentally but had not been documented previously.

The assumption of work- and stretchinduced hypertrophy of denervated fibres is not an entirely satisfactory explanation. If it was, then calf hypertrophy would be a much more common finding in longstanding $\mathrm{Sl}$ root lesions. The hypothesis might explain some of the effects on the calf muscles, which are the muscles most often used and stretched in the daily activities because of the upright posture of humans. Biopsy findings in two of our patients are compatible with this assumption and also with chronic denervation and reinnervation. ${ }^{38}$ However, the fact that in two of our patients the trapezius muscle and in another patient the anterior tibial muscle were hypertrophied weakens the hypothesis of combined work and stretch as a major cause and raises the question of further pathogenetic factors.

In our three patients dense continuing spontaneous discharges of complex potentials were the prominent finding in electromyography of the hypertrophied muscles. Lapresle et $a l{ }^{6}{ }^{6}$ Mielke and Ricker, ${ }^{9}$ Cooper et al,,$^{11}$ Lagueny et $a l,{ }^{14}$ Valenstein et $a l^{18}$ and Vasilescu et $a l^{19}$ also described striking spontaneous activity in the needle electromyography of the hypertrophied 
muscles denoted as "spontaneous motor unit potentials", "fasciculations", "myotonic", "pseudomyotonic", or "trains of repetitive" discharges.

Depending on firing rate and rhythm, different terms have been used to characterise complex discharges: "fasciculations" generally denote motor-unit type potentials firing at random. The terms "pseudomyotonic", "bizarre high frequency", or "complex repetitive" discharges have been used, when the potentials fire at a uniform frequency of 5 to $100 \mathrm{~Hz}$. The terms "grouped fasciculations" or "bizarre low frequency", "bizarre repetitive" and "myokymic" discharges have been used for more or less rhythmical firing at $5 \mathrm{~Hz}$ or less either of single complex potentials or of short bursts of potentials. Common to all these types of complex discharges is that they usually occur in chronic or longstanding neuromuscular disorders. High frequency complex repetitive discharges have been found in a variety of peripheral neurogenic disorders such as muscular dystrophy, polymyositis, spinal muscular atrophy, amyotrophic lateral sclerosis, and chronic neuropathies. ${ }^{40}$ Myokymic (low frequency) discharges and fasciculations have frequently been found in partial old nerve lesions such as chronic entrapment, ${ }^{41}{ }^{42}$ mechanical nerve injury, ${ }^{39}$ radiationinduced ${ }^{42-44}$ or inflammatory neuropathy, ${ }^{18194245}$ and hereditary neuropathy with liability to pressure palsy. ${ }^{45}$ The polyphasic or serrated action potentials of complex discharges are generated by groups of functionally linked muscle fibres, whereas fibrillations and sharp positive waves typical of acute denervation are generated by single denervated muscle fibres. The site of origin of the ectopic impulses causing the complex discharges is as yet uncertain and may not be the same in all types. While (high frequency) complex repetitive discharges have been shown to arise in the partially denervated or diseased muscle itself $^{46}$ myokymic discharges and fasciculations may arise in nerve fibres, and both, the actual locus of the causal nerve lesion ${ }^{42} 43$ as well as distal nerve sprouts ${ }^{48}$ have been proposed as possible sites of origin.

Electrical muscle activity normally reflects muscle fibre contraction. Therefore, when occurring excessively in a muscle, continuous spontaneous muscle activity may well give rise to hypertrophy, regardless of the anatomical location and function of the muscle. We therefore propose that the muscle hypertrophy was caused by the spontaneous activity. Such a pathogenesis has also been postulated in muscle hypertrophy of polyneuropathies with "pseudomyotonia". 1933 Similarly, Valenstein et $a l^{18}$ noted that a patient with chronic recurrent polyneuropathy showed the most spontaneous activity in those muscles which were hypertrophied. It could be assumed that the considerably hypertrophic muscle fibres in our biopsies were the ones which produced the spontaneous activity. It is interesting that in our specimen these were predominantly "tonic" type I fibres.

The clinician must be aware that isolated muscle hypertrophy can be a sign of partial peripheral nerve or root lesion, and it probably may occur in any skeletal muscle. Since neurogenic muscle hypertrophy is rare, focal myositis or primary muscle neoplasms might be suspected. This may lead to unnecessary, costly, and sometimes invasive investigations which could be avoided when the possibility of neurogenic hypertrophy is considered. The electromyographic finding in a hypertrophied muscle of profuse spontaneous activity confirms the diagnosis.

We thank Mrs Th Lauterburg and Mrs U Walker for technical assistance.

1 Graves RJ. Clinical lectures on the practice of medicine, Vo 1, 2nd ed. Dublin: Fannin, 1848:498.

2 Lhermitte MJ. Hypertrophie des muscles de la jambe consécutive à une lésion du nerf sciatique par balle. Rev Neurol (Paris) 1918;26:56-8.

3 Lhermitte MJ. Sur l'Hypertrophie musculaire consécutive aux lésions graves des nerfs périphériques. Une observation nouvelle. Rev Neurol (Paris) 1918;25:529-30.

4 Lhermitte J, Albessard. L'hypertrophie musculaire de la jambe dans la névrite sciatique. Rev Neurol (Paris) 1932;39:78-90.

5 Coste F, Hervet E. Diagnostic d'un gros mollet. Revue $d u$ Rhumatisme 1942;9:69-72.

6 Lapresle J, Fardeau M, Said G. L'hypertrophie musculaire vraie secondaire à une atteinte nerveuse périphérique: Etude clinique et histologique d'une observation d'hypertrophie du mollet consécutive à une sciatique. $R e v$ Neurol (Paris) 1973;128:153-60.

7 McComas AJ. Neuromuscular function and disorders. London: Butterworth, 1977 .

8 Bernat JL, Ochoa JL. Muscle hypertrophy after partial denervation: a human case. J Neurol Neurosurg Psychiatry 1978;41:719-25.

9 Mielke U, Ricker K, Emser W, Boxler K. Unilateral cal enlargement following $\mathrm{S} 1$ radiculopathy. Muscle Nerve 1982;5:434-8.

10 Montagna P, Martinelli P, Rasi F, Cirignotta F, Govoni E Lugaresi E. Muscular hypertrophy after chronic radiculopathy. Arch Neurol 1984;41:397-8.

11 Cooper WH, Ringel SP, Treihaft MM, Hall KA. Calf enlargement from S-1 radiculopathy. Report of two cases. $J$ Neurosurg 1985;62:442-4.

12 Ono S, Kuroiwa Y, Miyajima H, Nishimura Y, Honda N. Unilateral hypertrophy of the calf muscle. $J$ Neurol 1985;232:180.

13 De Visser M, Verbeeten B Jr, Lyppens KCM. Pseudohypertrophy of the calf following $\mathrm{S} 1$ radiculopathy Neuroradiology 1986;28:279-80.

14 Lagueny A, Coquet M, Julien J, et al. Hypertrophie musculaire neurogène. Association à des activités électrophysiologiques anormales. Rev Neurol (Paris) 1987; 143:189-200

15 Ricker K, Rohkamm R, Moxley RT. Hypertrophy of the calf with S-1 radiculopathy. Arch Neurol 1988;45:660-4.

16 Pareyson D, Morandi L, Scaioli V, Marazzi R, Boiardi A Sghirlanzoni A. Neurogenic muscle hypertrophy. Report of two cases. J Neurol 1989;236:292-5.

17 Serratrice G, Pellissier JF, Marini JF, Valentin-Leccese P. Un cas de sciatique avec hypertrophie du mollet. Rev Neurol (Paris) 1989;145:474-7.

18 Valenstein EM, Watson RT, Parker JL. Myokymia, muscle hypertrophy and percussion "myotonia" in chronic hypertrophy and percussion "myotonia" in chron

19 Vasilescu C, Alexianu M, Dan A. Muscle hypertrophy and a syndrome of continuous motor unit activity in prednisone-responsive Guillain-Barré polyneuropathy. Neurol 1984;231:276-9.

20 Morpurgo B. Ueber Activitäts-Hypertrophie der willkürlichen Muskeln. Virchows Archiv für pathologische Anatomie und Physiologie 1897;150:522-54

21 Goldspink G, Howells KF. Work induced hypertrophy in exercised normal muscles of different ages and the reversibility of hypertrophy after cessation of exercise. $J$ Physio 1974;239:179-93.

22 Sola OM, Martin AW. Denervation hypertrophy and atrophy of the hemidiaphragm of the rat. Am J Physiol 1953;172:324-32.

23 Torbergsen $\mathrm{T}$. A family with dominant hereditary myotonia, muscular hypertrophy, and increased muscular irritability, distinct from myotonia congenita Thomsen. Acta Neurol Scandinav 1975;54:225-32.

24 Bouwsma G, Van Wijngarden GK. Spinal muscular atrophy and hypertrophy of the calves. J Neurol Sci atrophy and hyper

25 Pearn JH, Hudgson P. Anterior-horn cell degeneration and gross calf hypertrophy. Lancet 1978;ii: 1059-61.

26 D'Alessandro R, Montagna P, Govoni E, Pazzaglia P. Benign familial spinal muscular atrophy with hypertrophy Benign familial spinal muscular atrophy with
of the calves. Arch Neurol 1982;39:657-60. 27 O'Donnell PP, Leshner RT, Campbell WW. Hypertrophia 
musculorum vera in familial ataxia. Arch Neurol 1986; 43:146-7.

28 Alberca R, Rafel E, Castilla JM, Gil-Peralta A. Increased mechanical muscle irritability syndrome. Acta Neurol Scandinav 1980;62:250-4.

29 Schwartz O, Jampel RS. Congenital blepharophimosis associated with a unique generalized myopathy. Arch Ophthal 1962;68:52-7.

30 Taylor RG, Layzer RB, Davis HS, Fowler WM. Continuous muscle fiber activity in the Schwartz-Jampel syndrome. muscle fiber activity in the Schwartz-Jampel syndro

31 Klein I, Perker M, Shebert R, Ayyar RR, Levey GS Hypothyroidism presenting as muscle stiffness and pseudohypertrophy: Hoffmann's syndrome. Amer J Med $1981 ; 70: 891-4$.

32 Bertorini TE, Igarashi M. Postpoliomyelitis muscle pseudohypertrophy. Muscle Nerve 1985;8:644-9.

$33 \mathrm{Krabbe} \mathrm{KH}$. The myotonia acquisita in relation to the post neuritic hypertrophies. Brain 1934;57:184-94.

34 Patel AN, Swami RK. Muscle percussion and neostigmine test in the clinical evaluation of neuromuscular disorders. N Engl J Med 1969;281:523-6.

35 Korczyn AD, Kuritzky A, Sandbank U. Muscle hypertrophy with neuropathy. J Neurol Sci 1978;44:275-9.

36 Stewart DM, Sola OM, Martin AW. Hypertrophy as a response to denervation in skeletal muscle. Zeitschrift für response to denervation in skeletal muscle.
vergleichende Physiologie 1972;76:146-67.

37 Mumenthaler $M$. Neurologische differentialdiagnose, 3rd ed. Stuttgart: Georg Thieme Verlag, 1988.

38 Drachman DB, Murphy SR, Nigam MP, Hills JR "Myopathic" changes in chronically denervated muscle. Arch Neurol 1967;16:14-24.
39 Medına JL, Chokroverty S, Reyes M. Localized myokymia caused by peripheral nerve injury. Arch Neurol 1976; 33:587-8.

40 Emeryk B, Hausmanova-Petrusewicz J, Nowak T. Spontaneous volley of bizarre high frequency potentials in neuromuscular diseases. Electromyogr Clin Neurophysiol 1974;14:303-12.

41 Stoehr M. Low frequency bizarre discharges. A particular type of electromyographical spontaneous activity in paretic skeletal muscle. Electromyogr Clin Neurophysiol

42 Albers JW, Allen AA, Bastron JA, Daube JR. Limb myokymia. Muscle Nerve 1981;4:494-504.

43 Stoehr M. Special types of spontaneous electrical activity in radiogenic nerve injuries. Muscle Nerve 1982;5:S78-83.

44 Harper CM, Thomas JE, Cascino TL, Litchy WJ. Distinction between neoplastic and radiation-induced brachial plexopathy, with emphasis on the role of EMG. Neurology 1989;39:502-6.

45 Roth G, Magistris MR. Neuropathies with prolonged conduction block, single and grouped fasciculations, localized limb myokymia. Electroenceph Clin Neurophysiol 1987; 67:428-38.

46 Ricker K, Meinck HM. Discharge pattern and origin of "pseudomyotonic" (high frequency) discharge trains in "pseudomyotonic" (high frequency) discharge trains

47 Trontelj J, Stålberg E. Bizarre repetitive discharges recorded with single fibre EMG. J Neurol Neurosurg Psychiatry 983;46:310-16.

48 Conradi S, Grimby L, Lundemo G. Pathophysiology of fasciculations in ALS as studied by electromyography of single motor units. Muscle Nerve 1982;5:202-8. 